Military Technical College Kobry El-Kobbah

Cairo, Egypt

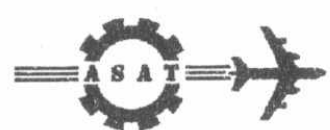

$10^{\text {th }}$ International Conference

On Aerospace Sciences\&

Aviation Technology

\title{
HYDROMAGNETIC STABILITY OF NON-STREAMING ANNULAR CYLINDRICAL INTERFACE
}

\author{
Ahmed E. Radwan and Hoda A. Hashem*
}

\begin{abstract}
The hydromagnetic stability of non-streaming bounded annular cylindrical interface having a tar mantle has been studied. The stability criterion has been derived, formulated in a dimensionless form and discussed. Some reported results have been obtained from the present general stability criterion as limiting cases. The longitudinal magnetic fields interior and exterior the fluid cylinder are stabilizing while the exterior transverse magnetic field is destabilizing. The different factors of the problem play an important role in stabilizing the model. As the Alfven wave velocity effect due to the magnetic field is much greater than that of the fluid speed, the destabilizing effect of the transverse field will be suppressed and stability sets in.
\end{abstract}

\section{KEY WORDS}

Stability, Hydromagnetic, and Cylindrical interface.

Mathematics Department, Faculty of Science, Ain Shams University, Cairo, Egypt.

" Department of Physics and Engineering Mathematics, Faculty of Engineering, AinShams University, Cairo Egypt. 


\section{INTRODUCTION}

The dynamical stability of a full circular fluid cylinder endowed with surface tension has been documented by Rayleigh [19]. An comprehensive account is given, with miscellaneous extensions, by Chandrasekhar [3] for that model as the fluid is incompressible and non-streaming whether it is subjected to the capillary force orland to the electromagnetic force with uniform axial magnetic field. This is due to its applications in many domains of science ranging from the spray to ink jet printers.

In the last decades and the present one, more attention paid for elaborating more comprehensive hydromagnetic stability problems, cf. refs. [5]-[23]. This is for its important applications in wide domains of science. E.g. the fluid jet amplifier and the electromagnetic ink jet printer and astrophysical problems concerning planetary and stellar atmosphere. However, not much work has been done in the MHD stability of flows with non-axisymmetric disturbances. Apart from the mathematical complexities arising in stability analysis with such disturbances as pointed out by Chandrasekhar (1981), there is an overriding reason for studying the response of system to these disturbances. In the absence of magnetic diffusion, non-axisymmetric disturbances generally twist the magnetic lines of force and produce an intimate coupling between the hydrodynamic and hydromagnetic influences that prevent the latter from being masked whatever is the small value of the magnetic field intensity. Axisymmetric disturbances, on the other hand, only bend the lines of force and under certain circumstances the hydromagnetic effects may dominate over those due to magnetic forces. Guided by this motivation, Kendall (1986) after his capillary experimental work attracted our wide attention for investigating the stability of annular jet.

Here we study the hydromgnetic stability of a non-streaming annular cylinder surrounded by a bounded tenuous medium.

\section{BASIC STATE}

We consider a non-streaming ideal annular fluid jet, we may write its velocity in the initial state in the form

$\underline{u}_{0}=(0,0,0)$

surrounded by a tenuous medium which is bounded by cylindrical barrier. Let $\mathrm{a}, \mathrm{R}$ and $b$ are the radii of the inner (tar cylinder), annular fluid and the tenuous cylindrical medium where $\mathrm{a}<\mathrm{R}<\mathrm{b}$ in the initial state. The tar medium of negligible motion occupies the region $0<r<a$, the streaming fluid occupies the region $a<r<R$ and the tenuous medium is being in the region $R<r<b$. The magnetized fluid is pervaded by the magnetic field

$\underline{\mathrm{H}}_{\mathrm{o}}=\left(0,0, \mathrm{H}_{0}\right)$

The tenuous medium is penetrated by the helical magnetic field 
$\underline{H}^{s}=\left(0, \xi R{ }_{0} H_{0} / r, \zeta H_{0}\right)$

where $\xi$ and $\zeta$ are real numbers while $\mathrm{H}_{0}$ is the intensity of the magnetic field. The components of $\underline{u}_{0}, \underline{H}_{0}$ and $\underline{H}_{0}^{s}$ are considered along the utilizing cylindrical coordinates $(r, \varphi, z)$ with $z$ - axis coinciding with the axis of the concentric cylinders. The system is acted by the inertia, capillary and electromagnetic forces, see fig. (1).

The basic equations are coming out from the combination of Maxwell electromagnetic equations together with the ordinary hydrodynamic equations and given as follows.

The hydromagnetic vector equation of motion:

$\rho \frac{\mathrm{d} \underline{\mathrm{u}}}{\mathrm{dt}}=-\nabla \mathrm{P}+\mu(\nabla \wedge \underline{\mathrm{H}}) \wedge \underline{H}$

with

$$
\begin{aligned}
& \frac{\mathrm{d}}{\mathrm{dt}}=\frac{\partial}{\partial \mathrm{t}}+(\underline{\mathrm{u}} \cdot \nabla) \\
& \nabla=\left(\frac{\partial}{\partial \mathrm{r}}, \frac{1}{\mathrm{r}} \frac{\partial}{\partial \varphi}, \frac{\partial}{\partial \mathrm{z}}\right)
\end{aligned}
$$

The equation of continuity :

$$
\nabla \cdot \underline{u}=0
$$

The equation expresses the conservation of flux

$$
\nabla \cdot \underline{H}=0
$$

The equation of magnetic field :

$$
\frac{\partial \underline{H}}{\partial t}=\nabla \wedge(\underline{u} \wedge \underline{H})
$$

The equation of the surface pressure $P_{S}$ due to the capillary force:

$$
P_{S}=\sigma\left(1 / r_{1}+1 / r_{2}\right)
$$

Here $\rho, \underline{u}$ and $P$ are the fluid mass density, velocity vector and kinetic pressure; $\mu$ and $\underline{H}$ are the magnetic field permeability coefficient and intensity, $\sigma$ the surface tension coefficient, $r_{1}$ and $r_{2}$ are the radii of curvature of the cylindrical fluid surface with

$$
1 / r_{1}+1 / r_{2}=\nabla \cdot \underline{N}
$$




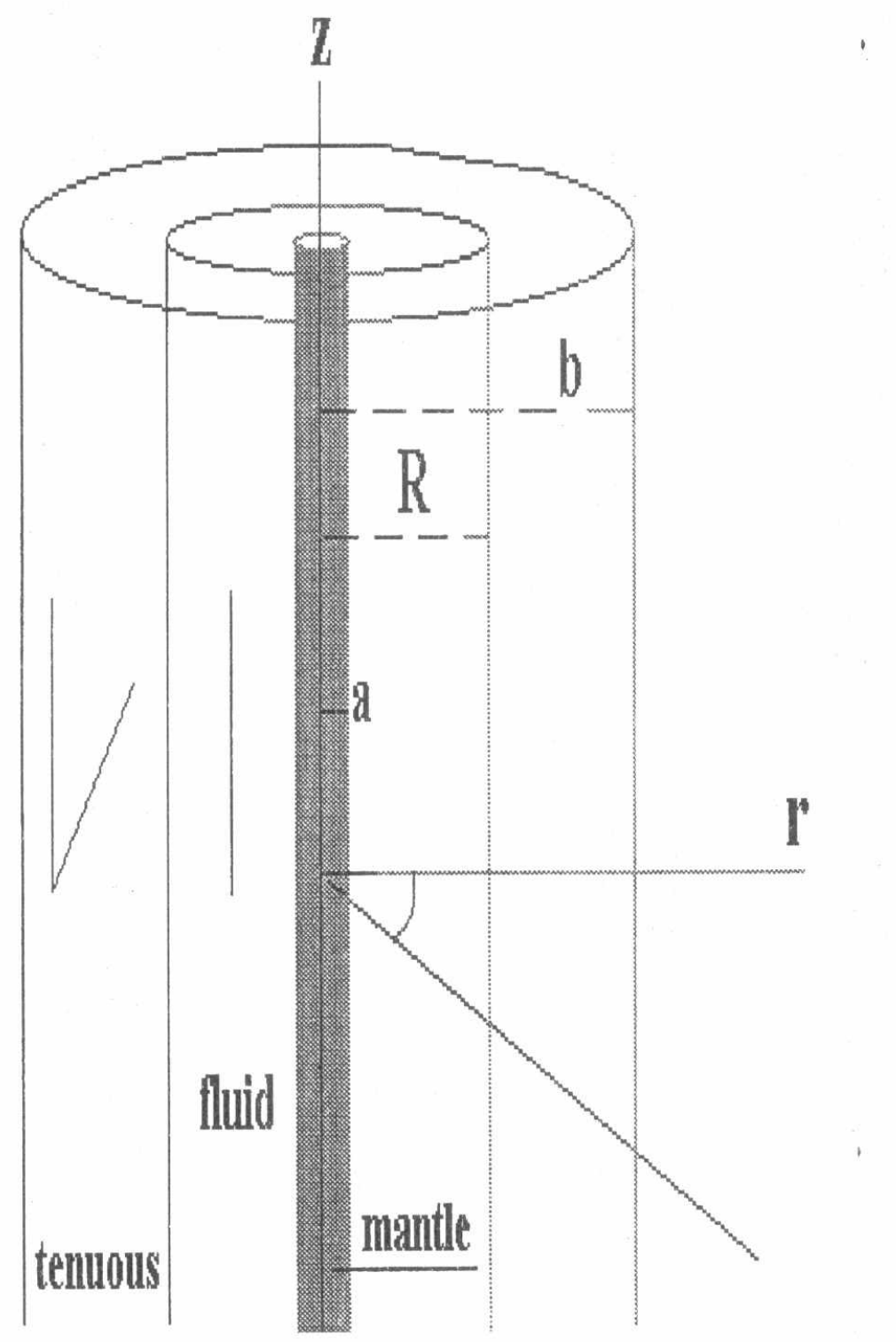

Fig. (1) Sketch for annular fluid jet 
The vector $\underline{\mathrm{N}}$ is, an outward unit vector normal to the surface of the annular fluid cylinder, being

$$
\underline{N}=\nabla f /|\nabla f \cdot \nabla f|^{1 / 2}
$$

with

$$
f(r, \varphi, z ; t)=0
$$

is the equation of the fluid boundary annular surface.

The basic equations concerning the tenuous medium in the region $R<r<b$ are given by

$$
\begin{aligned}
& \nabla \cdot \underline{H}^{\mathbf{s}}=0 \\
& \nabla \wedge \underline{H}^{\mathbf{s}}=0
\end{aligned}
$$

where $\underline{H}^{\mathbf{s}}$ is the intensity of the magnetic field with superscript $s$ characterizes variables concerning the behavior of the tenuous medium. Equation (14) expresses the conservation of flux while equation (15) comes out from Maxwell equations provided that there is no current flow exist.

The initial state as $t=0$ has been studied. The basic equations (4)----(13) are solved in the unperturbed state and it is found that

$$
\begin{aligned}
& P_{\text {os }}=\sigma / R \\
& P_{0} / \rho+\mu\left(\underline{H}_{0} \cdot \underline{H}_{0}\right) / 2 p=\text { const. }
\end{aligned}
$$

Due to the pressure balance at $r=R$, the kinetic pressure $P_{0}$ of the fluid is given by

$P_{0}=\sigma / R+\mu H_{0}{ }^{2} / 2\left(\zeta^{2}+\xi^{2}-1\right)$

Upon using the physical restriction that Po must be positive, we find $\zeta$ and $\xi$ must satisfay the restriction

$\zeta^{2}+\xi^{2} \geq\left(1-2 \sigma / \mu R H_{0}^{2}\right)$

where the equality corresponds to the limiting case of zero pressure and the coefficient of surface tension $\sigma$ and the intensity $H_{0}$ of the magnetic field are related by

$\sigma<(\mu / 2) \mathrm{R} \mathrm{H}_{0}{ }^{2}$ 


\section{PERTURBATION TECHNIQUE}

For small departures from the unperturbed state, every variable quantity $Q(r, \varphi, z, t)$ may be expressed as

$Q(r, \varphi, z, t)=Q_{0}(r)+\varepsilon(t) Q_{1}(r, \varphi, z)+\ldots$

where the suffices 0 and 1 characterize quantities in the non-perturbing and fluctuating states, respectively. Here $Q(r, \varphi, z, t)$ stands for $\underline{u}, \underline{P}, \underline{H}^{\mathbf{s}}, \underline{H}$ and the radial perturbed distance of the annular fluid cylinder. The parameter $\varepsilon(t)$ is the amplitude of the perturbation at an instant of time $t$.

$\varepsilon(t)=\varepsilon_{0} \exp (n t)$

with $\varepsilon_{0}(=\varepsilon$ at $t=0$ ) is the initial amplitude and $n$ is the growth rate or rather (if $n=i \omega$ is imaginary) the oscillation frequency. Suppose then that the perturbed interface is described by

$r=R+\eta, \quad \eta \ll R$

with

$\eta=\varepsilon(t) \exp [i(k z+m \varphi)]$

where $\mathrm{k}$ (any real number) is the longitudinal wave number along $z$-direction while $m$ (an integer) the azimuthal wave number. Here $\eta(\varphi, z, t)$ is the elevation of the free (annular fluid - tenuous) interface measured from the unperturbed position.

By the use of the expansion (21), the basic equations (4) --- (15) describing the motion of the annular tenuous fluids are linearized.

Since we seek a solution whose t-space dependence is given by $\exp [i(k z+m \varphi)$ $+n t]$ times an amplitude function of $r$. Based on the linear perturbation technique, every perturbed quantity $Q_{1}(r, \varphi, z, t)$ may be expressed as

$Q_{1}(r, \varphi, z, t)=Q_{1}(r) \exp [i(k z+m \varphi)+n t]$

By an appeal to the expansions (24) and (25), the relevant perturbation equations are simplified, solved and the non-singular solutions are identified.

These solutions must satisfy the following boundary conditions:

(i) The normal component of the velocity vector must be compatible with the velocity of the annular fluid-tenuous interface at $r=R$.

(ii) The radial component of the velocity vector must vanish across the cylindrical solid edge at $r=a$.

(iii) The jump of the normal component of the magnetic field across the annular fluid-tenuous interface must vanish at $r=R$. 
(iv) The radial component of the perturbed magnetic field in the tenuous medium must vanish at the cylindrical solid edge $r=b$.

Moreover as it is mentioned above, the present model is acted upon the capillary, inertia, fluid pressure gradient, fluid and tenuous electromagnetic forces. Here we have to apply the condition that the normal component of the total stresses must be continuous across the fluid-tenuous interface at $r=R$.

Consequently the following eigenvalue relation is established

$n^{2}=\frac{\sigma}{\rho R^{3}} \frac{x E_{1}}{E_{3}}\left(1-m^{2}-x^{2}\right)+\frac{\mu H_{o}^{2}}{\rho R^{2}}\left\{\xi^{2} \frac{x E_{1}}{E_{3}}+(m \xi+x \zeta)^{2} \frac{x E_{1} E_{4}}{E_{2} E_{3}}-x^{2}\right\}$

with

$$
\begin{aligned}
& E_{1}=I_{m}^{\prime}(x) K_{m}^{\prime}\left(q_{1} x\right)-I_{m}^{\prime}\left(q_{1} x\right) K_{m}^{\prime}(x) \\
& E_{2}=I_{m}^{\prime}\left(q_{2} x\right) K_{m}^{\prime}(x)-I_{m}^{\prime}(x) K_{m}^{\prime}\left(q_{2} x\right) \\
& E_{3}=I_{m}(x) K_{m}^{\prime}\left(q_{1} x\right)-I_{m}^{\prime}\left(q_{1} x\right) K_{m}(x) \\
& E_{4}=I_{m}^{\prime}\left(q_{2} x\right) K_{m}(x)-I_{m}(x) K_{m}^{\prime}\left(q_{2} x\right)
\end{aligned}
$$

Here $I_{m}$ and $K_{m}$ are the modified Bessel functions of the first and second kind of order $\mathrm{m}$ and the prime over them means derivative with respect to argument, and the different dimensionless wavenumbers of the present problem are being

$x=k R, a=q_{1} R, b=q_{2} R, k a=q_{1} x$

$k b=q_{2} x, 0<q_{1}<1,1<q_{2}<\infty$

\section{LIMITING CASES}

Upon discussing the relation (26), the hydromagnetic characteristics of the present model could be identified i.e. One may determine the instability domains and those of stability as well, and also the influence of the magnetic field on the capillary instability of the model. Moreover, we find the following. The argument of the modified Bessel functions occurred in the compound functions $\left(E_{1} / E_{3}\right)$ are $x$ and $q_{1} x$ but not $q_{2} x$ in the (contribution of the surface tension) general relation (26). This is logic because we have here annular fluid around a tar cylinder of radius $q_{1} R$ with $0<q_{1}<1$. In the second term of the right side of (26), concerning the magnetic field contribution, we see that the arguments of the modified Bessel functions $I_{m}, K_{m}$ and their derivatives of order $m$ are not only $x$ or $q_{1} x$ but also $q_{2} x$. In reality, this is due to the fact that the tenuous helical varying magnetic field is pervaded in a bounded region by cylindrical 
solid edge of radius $b\left(=q_{2} R\right)$ with $1<q<\infty$. Also, we note that the effects of the surface tension and the tenuous electromagnetic force depend on the kind of the (axisymmetric or non-axisymmetric) perturbation while that of the electromagnetic force acting upon the annular fluid is not depending on the kind of the perturbation.

Concerning (the pure hydrodynamic) more discussions of superposed fluids we may refer to Nayak \& Chakraborty (1984), Rosensweig (1985) and Miura (1987).

Since the present problem is somewhat more general, stability criteria of some problems of different models may be obtained from the general eigenvalue relation (26) as limiting cases with appropriate choices. In order to do so we have to write down about the behaviour of the modified Bessel functions. In view of the recurrence relations (Abramowitz (1970))

$2 I_{m}^{\prime}(x)=I_{m-1}(x)+I_{m+1}(x)$

$2 K_{m}^{\prime}(x)=-K_{m-1}(x)-K_{m+1}(x)$

and for each non-zero real value of $x$ that $I_{m}(x)$ is always positive and monotonic increasing while $\mathrm{K}_{\mathrm{m}}(\mathrm{x})$ is monotonic decreasing but never negative, we see that

$\mathrm{I}_{\mathrm{m}}^{\prime}(\mathrm{x})>0 \quad, \mathrm{~K}_{\mathrm{m}}^{\prime}(\mathrm{x})<0$

On the basis of these arguments, taking into account that $0 \leq q_{1}<1$ and $1<q_{2}<\infty$, we get

$$
\begin{aligned}
& \quad K_{m}\left(q_{2} x\right)<K_{m}(x)<K_{m}\left(q_{1} x\right) \\
& -\left|K_{m}^{\prime}\left(q_{2} x\right)\right|<-\left|K_{m}^{\prime}(x)\right|<-\left|K_{m}^{\prime}\left(q_{1} x\right)\right| \\
& I_{m}^{\prime}\left(q_{1} x\right)<I_{m}^{\prime}(x)<I_{m}^{\prime}\left(q_{2} x\right) \\
& I_{m}\left(q_{1} x\right)<I_{m}(x)<I_{m}\left(q_{2} x\right)
\end{aligned}
$$

It follows, on using (27) - (31) together with (34) - (37), for $x \neq 0$ that

$$
\begin{aligned}
& \frac{E_{1}}{E_{3}}=\frac{I_{m}^{\prime}(x)\left|K_{m}^{\prime}\left(q_{1} x\right)\right|-I_{m}^{\prime}\left(q_{1} x\right)\left|K_{m}^{\prime}(x)\right|}{I_{m}(x)\left|K_{m}^{\prime}\left(q_{1} x\right)\right|+I_{m}^{\prime}\left(q_{1} x\right) K_{m}(x)}=\frac{+\underline{v e}}{+\underline{v e}}>0 \\
& \frac{E_{4}}{E_{2}}=\frac{I_{m}(x)\left|K_{m}^{\prime}\left(q_{2} x\right)\right|+I_{m}^{\prime}\left(q_{2} x\right) K_{m}(x)}{I_{m}^{\prime}(x)\left|K_{m}^{\prime}\left(q_{2} x\right)-I_{m}^{\prime}\left(q_{2} x\right)\right| K_{m}^{\prime}(x) \mid}=\frac{+\underline{v e}}{-\underline{v e}}<0
\end{aligned}
$$

and consequently, for each real value of $x \neq 0$ we have 
$\frac{E_{1} E_{4}}{E_{2} E_{3}}<0$

Moreover on utilizing the limits

$$
\begin{array}{ll}
\lim _{q_{1} \rightarrow 0} K_{m}^{\prime}\left(q_{1} x\right) \rightarrow \infty, & \lim _{m} I_{m}^{\prime}\left(q_{2} x\right) \rightarrow \infty \\
& q_{2} \rightarrow \infty
\end{array}
$$

we may show that

$$
\begin{aligned}
& \lim _{q_{1} \rightarrow 0} \frac{E_{1}}{E_{3}}=\frac{I_{m}^{\prime}(x)}{I_{m}(x)} \\
& \lim _{q_{2} \rightarrow \infty} \frac{E_{4}}{E_{2}}=\frac{K_{m}(x)}{K_{m}^{\prime}(x)} \\
& \lim _{q_{1} \rightarrow 0 \text { and } q_{2} \rightarrow \infty} \frac{E_{1} E_{4}}{E_{2} E_{3}}=\frac{I_{m}^{\prime}(x) K_{m}(x)}{I_{m}(x) K_{m}^{\prime}(x)}
\end{aligned}
$$

Now, we may obtain the results of some published works from the present general results as limiting cases.

Consider $m=0, \xi=0, \zeta=0, H_{0}=0$ and $q_{1} \rightarrow 0$ whether $q_{2} \rightarrow \infty$ or not, the relation (26) yields

$$
n^{2}=\frac{\sigma}{\rho R^{3}} \frac{I_{1}(x)}{I_{0}(x)}\left(1-x^{2}\right)
$$

where we have used the limit $(43)$ and that $I_{o}^{\prime}(x)=I_{1}(x)$. The dispersion relation (48) coincides with that derived for first time by Rayleigh (1945) in investigating the breaking up of a fluid column endowed with surface tension. The discussions of the relation (48) reveal that the capillary cut off wavenumber is $x=1$ while the maximum growth rate of instability occurs at $x=0.697$.

For non-axisymmetric modes $m>0$, equation (26) gives

$$
n^{2}=\frac{\sigma}{\rho R^{3}} \frac{x I_{m}^{\prime}(x)}{I_{m}(x)}\left(1-m^{2}-x^{2}\right)
$$


which is the classical dispersion relation of a liquid cylinder in vacuum derived by Chandrasekhar (1981) on using the theoretical technique laid earlier by Rayleigh (1945).

If we put $\xi=0, \zeta=1, q_{1} \rightarrow 0, q_{2} \rightarrow \infty$ and $m=0$, the relation (26) reduces to

$$
n^{2}=\frac{\sigma}{\rho R^{3}} \frac{x I_{1}(x)}{I_{0}(x)}\left(1-x^{2}\right)-\frac{\mu H_{0}^{2}}{\rho R^{2}} \frac{x}{I_{0}(x) K_{1}(x)}
$$

This relation coincides with Chandrasekhar's dispersion relation (1981) Chapt. XII obtained in examining the effect of an axial magnetic field on the axisymmetic capillary instability of a full fluid cylinder.

If we put $\xi=0, \zeta=1, \mathrm{q}_{1} \rightarrow 0, \mathrm{q}_{2} \rightarrow \infty$ while $\mathrm{m} \geq 0$ in equation (26), and using (34) (47), we get

$$
n^{2}=\frac{\sigma}{\rho R^{3}} \frac{x I_{m}^{\prime}(x)}{I_{m}(x)}\left(1-m^{2}-x^{2}\right)+\frac{\mu H_{0}^{2}}{\rho R^{2}} \frac{x}{I_{m}(x) K_{m}^{\prime}(x)}
$$

where equation (50) could be obtained from (51) for axisymmetric perturbation.

If we set $q_{2} \rightarrow \infty, \xi=0, \quad \zeta=0, H_{0}=0$, and $m \geq 0$ where $0<q_{1}<1$ in equation (26), we obtain

$$
n^{2}=\frac{\sigma}{\rho R^{3}} \frac{x E_{1}}{E_{3}}\left(1-m^{2}-x^{2}\right)
$$

This relation has been derived for first time by Radwan (1987), on utilizing the lagrangian energy principle, in investigating the capillary instability of a fluid cylinder having a regular solid cylinder as a mantle.

The results of Malik and Singh (1991) could be recovered from the present general results as limiting case. However, in dealing with the mathematical derivation of Malik and Singh (1991), it is found that the electromagnetic term in equation (26) there is wrong. This can be shown explicitly if we put there $\mu_{1}=\mu_{2}=\mu$, then the results in right side of equation (26) there must be as given here in equations (50) and (51) and as given and documented by Chandrasekhar (1981) as $m=0$.

\section{HYDRODYNAMIC STABILITY DISCUSSIONS}

In absence of the electromagnetic forces the relation (26) reduces to 


$$
n^{2}=\frac{\sigma}{\rho R^{3}} \frac{x\left[I_{m}^{\prime}(x) K_{m}^{\prime}\left(q_{1} x\right)-I_{m}^{\prime}\left(q_{1} x\right) K_{m}^{\prime}(x)\right]}{\left(I_{m}(x) K_{m}^{\prime}\left(q_{1} x\right)-I_{m}^{\prime}\left(q_{1} x\right) K_{m}(x)\right)}\left(1-m^{2}-x^{2}\right)
$$

In view of the recurrence relations (32) and (33) and the inequalities (34) - (40) as the annular fluid is stationary. The discussions of the relation (54) reveal that the behavior of the non-dimension growth rate $n / \sqrt{\sigma /\left(\rho R^{3}\right)}$ is mainly depending on the quantity $\left(1-m^{2}-x^{2}\right)$ since the associated fraction is positive for each non-zero real values of $x$. Taking into account the inequality (40), equation (54) produce for all values of $0<q_{1}<1$ that: (i) $n^{2}(\sigma)<0$ for all $m \neq 0$; (ii) $n^{2}(\sigma)>0$ for $-1<x<1$ and $n^{2}(\sigma)$ $\leq 0$ for $x \geq 1$ or $x \leq-1$ for $m=0$. This means that the stability domains are $1<x<\infty$ as $m=0$ and $0<x<\infty$ as $m \geq 1$. While the only unstable domain is $0<x<1$ as $m=0$. The marginal stability case which is a transition from stability states to those of instability is given as $\mathrm{x}=0$.

We conclude that the non-streaming annular fluid cylinder is capillary stable to all non-axisymmetric modes and it is so only to axisymmetric (sausage) mode whose wavelength $\lambda=2 \pi / k$ shorter than the circumference $2 \pi R$ of the annular cylinder, while it is unstable to sausage mode whose $\lambda$ is longer than $2 \pi R$. Clearly if $\lambda=2 \pi R$ in the sausage mode, we get neutral stability.

All the foregoing results are valid for all values of $q_{1}$ such that $0<q_{1}<1$. The numerical analysis of the stability criterion (54) for different values of the problem's parameters for $m=0$, reveal that the maximum mode of instability obtained for the growth rate in the unstable domain $0 \leq x<1$ is occurred as $q_{1}=0$. The unstable domains are decreasing with increasing $q_{1}\left(0<q_{1}<1\right)$ values. This means that the existing of the tar mantle inside the annular fluid has the effect of shrinking the unstable regions for different values of $q_{1}$. Thus $q_{1}$ has a stabilizing influence. However, it is found that there is always unstable domain whatever is the largest value of $q_{1}$ with $0<q_{1}<1$ and that all the unstable regions (with different maximum temporal amplification) have the unstable domain $0<x<1$.

\section{MAGNETODYNAMIC STABILITY DISCUSSIONS}

In absence of the capillary force and assuming that the annular fluid model is acted upon the electromagnetic, inertia and pressure gradient forces, the general relation (26) yields

$$
\begin{aligned}
n^{2}=\frac{\mu H_{0}^{2}}{\rho R^{2}}\{- & x^{2}+\frac{x\left[I_{m}^{\prime}(x) K_{m}^{\prime}\left(q_{1} x\right)-I_{m}^{\prime}\left(q_{1} x\right) K_{m}^{\prime}(x)\right]}{\left[I_{m}(x) K_{m}^{\prime}\left(q_{1} x\right)-I_{m}^{\prime}\left(q_{1} x\right) K_{m}(x)\right.} \times \\
& {\left.\left[\xi^{2}+(m \xi+x \zeta)^{2} \frac{\left(I_{m}^{\prime}\left(q_{2} x\right) K_{m}(x)-I_{m}(x) K_{m}^{\prime}\left(q_{2}(x)\right.\right.}{\left(I_{m}^{\prime}\left(q_{2} x\right) K_{m}^{\prime}(x)-I_{m}^{\prime}(x) K_{m}^{\prime}\left(q_{2}(x)\right)\right.}\right]\right\} }
\end{aligned}
$$


The effect of the axial magnetic field penetrated in the annular fluid is represented by the term $\left(-x^{2}\right)$ following the quantity $\left(\mu \mathrm{H}_{0}^{2} / \rho \mathrm{R}_{0}^{2}\right)$. It has always a stabilizing effect, and that effect is valid for all parameters of the problem iand it is independent of the kind of the perturbation. The influence of the tenuous axial magnetic field is represented by the terms including $\zeta$ in the relation (55) as $\xi=0$, viz.,

$x^{2} \zeta^{2} \frac{\left(I_{m}(x) K_{m}^{\prime}(q x)-I_{m}^{\prime}(q x) K_{m}^{\prime}(x)\right)\left(I_{m}^{\prime}\left(\mathrm{q}^{\prime} x\right) K_{m}(x)-I_{m}(x) K_{m}^{\prime}(q x)\right)}{\left(I_{m}(x) K_{m}^{\prime}(q x)-I_{m}^{\prime}(q x) K_{m}(x)\right)\left(\dot{I}_{m}^{\prime}(q x) K_{m}^{\prime}(x)-I_{m}^{\prime}(x) K_{m}^{\prime}(q x)\right)}$

From the viewpoint of the recurrence relations (32) and (33) and the inequality (42), noting that the sign of $\zeta$ is immaterial in the expression (56), we found that the longitudinal tenuous magnetic field has always a stabilizing effect. That effect is valid not only in the axisymmetric disturbance but also in those of non-axisymmetric disturbance but also in those of non-axisymmetric. We expect here also that both the geometrical factors $q_{1}$ and $q_{2}$ will increase such stabilizing influence. It is worth while to mention here also that this stabilizing effect is due to the existing of the quantity $E_{1} E_{4} / E_{2} E_{3}$ since $E_{4} / E_{2}<0$ while $E_{1} / E_{3}>0$, see the inequalities (40) - (42).

The effect of the transverse magnetic field is represented by the terms including $\xi$ :

$$
\xi^{2} \frac{x E_{1}}{E_{3}}+m^{2} \xi^{2} \frac{x E_{1} E_{4}}{E_{2} E_{3}}
$$

For $m=0$, we see that its effect is represented by the term $\xi^{2} \times\left(E_{1} / E_{3}\right)$. In view of $(40)$ we find that it has a pure destabilizing effect for all short and long wavelengths. In the non-axisymmetric disturbance $m \geq 1$, taking into account the inequalities (40) - (42), it has a destabilizing effect due to the term $x \xi^{2}\left(E_{1} / E_{3}\right)$ while it has a stabilizing effect due to the term $x m^{2} \xi^{2}\left(E_{1} E_{4}\right) /\left(E_{2} E_{3}\right)$. Therefore, the transverse magnetic field is stabilizing if

$$
m^{2} E_{4}>E_{2}
$$

There is a common effect due to both the longitudinal and transverse magnetic fields represented by the term $2 m \xi \zeta x^{2}\left(E_{1} E_{4}\right) /\left(E_{2} E_{3}\right)$. It has no effect in $m=0$ disturbance but it is stabilizing in the non-axisymmetric disturbance $m \geq 1$.

To sum up, the electromagnetic force acting on the annular fluid is stabilizing, while the acting magnetic field in the tenuous medium is stabilizing or destabilizing according to certain restrictions.

\section{HYDROMAGNETIC STABILITY DISCUSSIONS}

This case is the general one in which the annular fluid together with the tenuous medium coaxial between two circular cylindrical interfaces is subject to the combined 
effects of the capillary, inertia, pressure gradient and electromagnetic forces. The stability criterion of such case is given by equation (26) in its general form.

The capillary force is stabilizing in the domains $(0<x<\infty$ in $m \geq 1$ perturbations) and ( $1 \leq x<\infty$ in $m=0$ perturbations) while it is destabilizing in the domains $(0 \leq x<1$ in $m=0$ perturbations) for all values of $q_{1}$ with $0<q_{1}<\infty$. The electromagnetic force due to the longitudinal magnetic field pervaded in the fluid has stabilizing effect and that influence is independent of the kind of the perturbation. The electromagnetic force due to the helical tenuous varying magnetic fields has different effects which are depending on the kind of perturbation and on $\xi$ and $\zeta$ values. The tenuous longitudinal magnetic field is represented by terms including $\zeta$ while the transverse field is represented by terms including $\xi$. The term $\times \xi^{2} E_{1} / E_{3}$ is always positive (for $x>0)$ as explained before, thus it is always destabilizing. The term $x(m \xi+x \xi)^{2} E_{1} E_{4} /\left(E_{2} E_{3}\right)$ is always negative and thus it is stabilizing. However, if $m$ $x \zeta \xi$ is negative, then $(m \xi+x \zeta)^{2}$ is smaller than otherwise and so it causes less stabilizing. We have the choice of the sign of $m$. If we consider $m$ so that $m \times \zeta \xi$ is negative, (e.g. by reversing the perturbed tenuous helical magnetic field) the stabilization character will be decreased. Since the stability is determined by the least stable mode we have essentially to deal with the case $m \times \zeta \xi<0$. It is remarkable that the stabilizing influence of the term $x(m \xi+x \zeta)^{2} E_{1} E_{4} /\left(E_{2} E_{3}\right)$ is disappeared and suppressed when $m \xi+x \zeta=0$. In such case we observe that $n^{2}$ is then independent of $\zeta$ and only the destabilizing effect of the transverse tenuous magnetic field remains through the term $\times \xi^{2} E_{1} / E_{3}$.

We hope in near future to determine the effect of the streaming on the stability of this model. 


\section{REFERENCES}

[1] Abramowitz, M. and Stegun, I., "Handbook of Mathematical Functions" (Dover Publ., N.Y., 1970).

[2] Chhabra, R.K. and Trehan, S.K., Astrophy. J., 158 (1989) 67.

[3] Chandrasekhar, S., "Hydrodynamic and Hydromagnetic Stability" (Dover Publ., N.Y., 1981).

[4] Kendall, J.M., Phys. Fluids, 29 (1986) 2086.

[5] Lee, C.P. and Wang, T.G., Phys. Fluids, $\underline{6}$ (1989) 967.

[6] Lee J.G. and Chen, L.D., American Inst. of Aeronautics and Astronautics J. (AIAA), 29 (1991) 1589.

[7] Malik, S.K. and Singh, M., Int. J. Engng. Sci., $\underline{29}$ (1991) 1493.

[8] Miura, A., J. Geophys. Res., 92 (1987) 3195.

[9] Nayak, A. and Chakraborty, B., Phys. Fluids, 27 (1984) 1937.

[10] Osherovich, V.A., Solar Phys., $\underline{77}$ (1982) 63.

[11] Osherovich, V.A., Astrophys. J., 933 (1983) 221.

[12] Radwan A., Elazab S. and Hydia W., Phys. Script., 50 (1994)142.

[13] Radwan, A.E., Phys. Script., 56 (1997) 61.

[14] Radwan A.E., Tamkang J. Math., 28 (1997) 127.

[15] Radwan A.E., Int. J. Engng. Sci., 37 (1999) 123.

[16] Radwan et al., Chaos, Solitons \& Fractals, 12 (2001) 1729.

[17] Radwan A.E., Sileem S.M. and Ashour A.A., Ind. J. Maths., 43 (2001) 1.

[18] Radwan A.E. and Hashem H.A., Nuovo Cimento (2002) in press.

[19] Rayleigh, J.W. ( $3^{\text {rd }}$ Lord), "The theory of Sound Waves" (Dover publ., N.Y., 1945).

[20] Rivat, P. \& Etay, J. and Garnier, M., Europ. J. Mech., B/Fluids, 10 (1991) 537.

[21] Rosensweig, A.E., 'Ferrohydrodynamics" (Cambridge, N.Y., 1985).

[22] Sharma, K.C., Astrophys. J., 94 (1983) 419.

[23] Tsinganos, K.C.; Astrophys. J., 252 (1982) 775. 